\title{
Brain Systems Mediating Cognitive Interference by Emotional Distraction
}

\author{
Florin Dolcos ${ }^{1}$ and Gregory McCarthy ${ }^{1,2}$ \\ ${ }^{1}$ Duke University-University of North Carolina Brain Imaging and Analysis Center, Duke University, Durham, North Carolina 27710, and ${ }^{2}$ Department of \\ Veterans Affairs Medical Center, Durham, North Carolina 27705
}

\begin{abstract}
Flexible behavior depends on our ability to cope with distracting stimuli that can interfere with the attainment of goals. Emotional distracters can be particularly disruptive to goal-oriented behavior, but the neural systems through which these detrimental effects are mediated are not known. We used event-related functional magnetic resonance imaging to investigate the effect of emotional and nonemotional distracters on a delayed-response working memory (WM) task. As expected, this task evoked robust activity during the delay period in typical WM regions (dorsolateral prefrontal cortex and lateral parietal cortex). Presentation of emotional distracters during the delay interval evoked strong activity in typical emotional processing regions (amygdala and ventrolateral prefrontal cortex) while simultaneously evoking relative deactivation of the WM regions and impairing WM performance. These results provide the first direct evidence that the detrimental effect of emotional distracters on ongoing cognitive processes entails the interaction between a dorsal neural system associated with "cold" executive processing and a ventral system associated with "hot" emotional processing.
\end{abstract}

Key words: affect; arousal; learning and memory; emotional interference; emotional-cognitive interaction; affective disorders

\section{Introduction}

Goal-directed behavior depends on executive processes, such as working memory (WM), which allows one to maintain and manipulate information relevant to our current tasks over short periods of time (Baddeley, 1986). Distraction challenges our ability to maintain focus on goal-relevant information, and emotional stimuli are particularly potent distracters that can capture attention and reallocate processing resources (Ellis and Ashbrook, 1988) and thus impair cognitive performance. The neural systems that mediate these detrimental effects of emotion on goal-directed cognitive processing are not known. Understanding the mechanisms by which emotion impairs cognitive functions has important implications for affective disorders, such as depression and posttraumatic stress disorder, which are characterized by increased susceptibility to emotional distraction. Here, we report an investigation of the brain systems involved when task-irrelevant emotional stimuli interfere with the performance of a working memory task.

Models of affective-cognitive interactions inspired by clinical investigations suggest that increased emotional distractibility and

\footnotetext{
Received Sept. 10, 2005; revised Jan. 8, 2006; accepted Jan. 9, 2006.

This work was supported by National Institutes of Health Grants P01-NS41328, P50-MH60451, and R01MH05286. G.M. was supported by a Department of Veterans Affairs (DVA) Senior Research Career Scientist Award and by the DVA Mental IIIness Research, Education, and Clinical Center. We thank Jordan Tozer, Susan Music, and Jessica Aitken for assistance with data collection; Philip Kragel, Kenny Tao, Deepak Bastakoty, and Mary Beth Nebel for assistance with data analysis and manuscript preparation; and Drs. Roberto Cabeza, Scott Huettel, Kevin Pelphrey, Jamie Morris, Gunes Yucel, and Lihong Wang for comments on a previous version of this manuscript.

Correspondence should be addressed to Dr. Gregory McCarthy, Duke University-University of North Carolina Brain Imaging and Analysis Center, 163 Bell Building, Box 3918, Durham, NC 27710. E-mail: gregory.mccarthy@duke.edu.

D0I:10.1523/JNEUROSC1.5042-05.2006

Copyright $\odot 2006$ Society for Neuroscience $\quad$ 0270-6474/06/262072-08\$15.00/0
}

impaired ability to maintain focus on relevant information observed in depressed patients (Watts et al., 1988) are the consequences of altered interactions between a dorsal brain system involved in "cold" executive processing and a ventral system involved in "hot" emotional processing (Mayberg, 1997; Drevets and Raichle, 1998). The dorsal executive system includes dorsolateral prefrontal cortex (dlPFC) and lateral parietal cortex (LPC), which are critical to active maintenance of goal-relevant information in WM, and increased activity in these regions during WM tasks is typically associated with increased performance (Courtney et al., 1997; Fuster, 1997; Smith and Jonides, 1999; Chafee and Goldman-Rakic, 2000). The ventral affective system includes brain regions involved in emotional processing, such as the amygdala, ventrolateral PFC (vlPFC), and medial PFC (Davidson and Irwin, 1999; Davis and Whalen, 2001; Phan et al., 2002; Zald, 2003).

Although evidence from clinical and nonclinical populations (Mayberg, 1997; Drevets and Raichle, 1998; Yamasaki et al., 2002; Wang et al., 2005) suggests that cognitive and affective aspects of processing evoke different patterns of brain activity in these two neural systems, direct evidence that an interaction between these systems causes an impairment of functions that are dependent on the dorsal executive system is lacking.

The present study addressed this issue by using functional magnetic resonance imaging (fMRI) to investigate the effect of emotional distracters on working memory maintenance. Healthy participants performed a delayed-response WM task for faces, with emotional or nonemotional novel stimuli presented as distracters during the delay interval. We made two predictions. First, on the basis of evidence concerning the interfering effect of emotion on cognitive functions (Seibert and Ellis, 1991; Oaksford et al., 1996), we predicted that emotional distracters would have the 


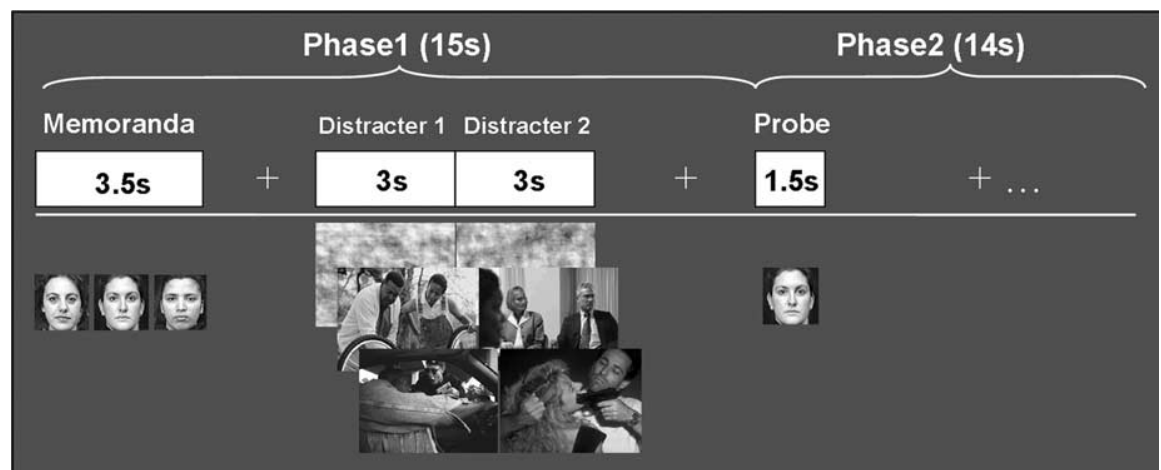

Figure 1. Diagram of the working memory task showing the event order and trial types. Three categories of trials, defined by the type of distracters presented during the delay interval, were involved. Each trial contained two distracters of the same type, which were consecutively presented for 3 s. Subjects were instructed to encode and maintain the memoranda into working memory, look at the distracters, and then indicate by pressing a response button whether the probes were part of the memoranda (1, yes; 2, no).

most detrimental effect on WM performance. Second, on the basis of evidence suggesting dissociable effects of emotion on brain activity (Mayberg, 1997), and consistent with the expected behavioral effect, we predicted that emotional distracters would increase activity in brain regions responsible for emotional processing (ventral system) while simultaneously decreasing activity in brain regions responsible for active maintenance of taskrelevant information into WM (dorsal system).

\section{Materials and Methods}

Subjects. Eighteen healthy right-handed young adults participated in the study. Data from three subjects were excluded from analyses because of mechanical failures in recording the behavioral responses (in two subjects) and because of low memory performance (in one subject). Thus, analyses were performed on data from 15 subjects (18-31 years of age; average age, $22 ; \mathrm{SD}=3.8$ ). Because other investigators have reported gender differences in the processing of emotional information (Shields, 1991; Lang et al., 1993), we restricted our study to female participants to maintain homogeneity of the subject sample. The experimental protocol was approved for ethical treatment of human participants by the Institutional Review Board at Duke University Medical Center.

Stimuli. Subjects performed a delayed-response WM task with novel distracters presented during the delay interval (Fig. 1). The memoranda consisted of sets of three human faces ( $50 \%$ females $/ 50 \%$ males), and the distracters consisted of pictures depicting emotional scenes, pictures depicting neutral scenes, and digitally scrambled versions of these pictures. The scrambled pictures had the same average spatial frequency and luminance as the meaningful pictures, and thus served as a no-distraction perceptual control. The emotional and nonemotional pictures were selected from the International Affective Picture System (IAPS) (Lang et al., 1997) and were supplemented by in-house sources used in previous studies (Yamasaki et al., 2002) to equate emotional and neutral pictures for complexity and human presence. Emotional distracters consisted of high-arousing and negative pictures (e.g., aggressive behavior, mutilations), and neutral distracters consisted of low-arousing and neutral pictures (e.g., mundane activities). The IAPS means in the 1-9 arousal and valence scales, respectively, were as follows: $6.2 / 2.0$, for negative pictures, and 3.5/5.2 for neutral pictures. All emotional and neutral pictures contained human characters, and both memoranda and distracters were presented in color. A total of 120 experimental trials, identified based on the type of distracter ( 40 emotional, 40 neutral, and 40 scrambled), were involved.

Experimental procedures. The pool of 120 trials was divided into 10 sets of 12 trials (four emotional, four neutral, and four scrambled), which were randomly assigned to 10 study blocks. Ten different block orders were randomly assigned to the participants. To avoid induction of long- lasting mood states, the trials within each block were pseudorandomized so that no more than three trials of the same type were consecutively presented. The task involved a two-phase design. As illustrated in Figure 1, phase 1 of each trial started with the presentation of a memorandum (3.5 s.), which subjects encoded and maintained in WM for $11.5 \mathrm{~s}$. A delay of $2 \mathrm{~s}$ followed the offset of the memoranda, and then two distracters of the same category were presented consecutively for a total time of $6 \mathrm{~s}(3 \mathrm{~s}$ each). The subjects were instructed to look at the distracters but maintain focus on the WM task. The beginning of phase 2 coincided with the presentation of a single-face probe $(1.5 \mathrm{~s})$. The subject's task was to indicate whether the probe was one of the three-face memorandum or a new face (half of the probes were old, and half were new). Subjects were instructed to make quick and accurate responses while the probes were on the screen. Each probe was followed by a $12.5 \mathrm{~s}$ fixation interval to allow the hemodynamic response to return to the baseline. The total length of each trial was $29 \mathrm{~s}$.

After scanning, subjects performed two consecutive rating tasks, in which they assessed the meaningful distracters (i.e., the emotional and neutral pictures) for distractibility (as perceived during the WM task) and emotional intensity, using a four-point Likert scale (1, lowest; 4, highest). Based on these ratings, individual indices of distractibility and emotional reactivity were calculated by averaging each subject's ratings of the meaningful distracters. These two measures were assessed to confirm that perceived distractibility and emotional intensity covary with each other and to directly link behavioral responses with brain activity. For instance, the emotional intensity ratings were used to separate emotional and neutral distracters according to subjects' own responses to each picture.

Imaging protocol. MR scanning was conducted on a 4T GE scanner (General Electric, Milwaukee, WI). After localizer, anatomical series, and high-order shimming, a series of 30 functional slices were acquired (axial plane; echo time, $31 \mathrm{~ms}$; field of view, $25.6 \mathrm{~cm}^{2}$ ) using an inverse-spiral pulse sequence. Functional voxels were isotropic $\left(4 \mathrm{~mm}^{3}\right)$, and the acquisition repetition time (TR) was $2 \mathrm{~s}$, allowing full-brain coverage. High-resolution three-dimensional spin-echo structural images were acquired for each functional slice in axial orientation (ratio, 4:1, in-plane resolution, $1 \mathrm{~mm}^{2}$ ).

Data analysis. Statistical analyses were preceded by the following preprocessing steps performed with custom Brain Imaging and Analysis Center tools: quality assurance, TR alignment, motion correction, coregistration, normalization, and smoothing $\left(8 \mathrm{~mm}^{3}\right.$ kernel). Data analysis used voxel-wise and region of interest (ROI) analyses to compare brain activity associated with the conditions of interest (e.g., emotional vs neutral scene distracters). For individual analyses, the fMRI signal was selectively averaged in each subject as a function of trial type (i.e., emotional, neutral, and scrambled scene) and time point (one prestimulus and 13 poststimulus onset time points), using custom BIAC software, and pairwise $t$ statistics for the contrast of interest (e.g., emotional vs neutral) were calculated for each subject. Individual analyses produced wholebrain average and activation $t$ maps, for each condition, contrast of interest, and time point. Unless otherwise specified, only correct trials were included in the analyses. Also, no assumption was made about the shape of the hemodynamic response function.

The outputs of individual analyses were used as inputs for second-level random-effects group analyses. The group analyses involved both voxeland ROI-based statistics (i.e., $t$ tests/ANOVAs and post hoc analyses performed with Fisher's PLSD), which were performed using the individual activation $t$ maps and percent signal change data extracted from functional ROIs. The ROIs were traced around the voxels showing the maximum effects in the contrasts of interest (the average ROIs size was 39 contiguous voxels), as identified by the voxel-based analyses. As a general 
rule, voxel wise analyses were performed on changes in MR units from a prestimulus baseline, whereas ROI analyses were performed on percentage signal changes from baseline. Unless otherwise specified, for both voxel- and ROI-based analyses, an intensity threshold of $p<0.001(t>3.79)$ and an extent threshold of 10 contiguous voxels were used.

Finally, to investigate individual differences concerning the brain-behavior relationship in the presence of emotional distraction, acrosssubject voxel-based correlation analyses were performed between fMRI data from brain regions showing greater group effects of emotion (e.g., right vlPFC) and behavioral measures (i.e., distractibility/emotional reactivity ratings and memory performance). For all analyses, the results of statistics performed on the fMRI signal from the delay peak time points (i.e., the time points of the delay period showing the highest statistical significance for the contrasts of interest) are reported.

\section{Results}

\section{Behavioral results}

Emotional intensity and distractibility ratings

The average scores for emotional intensity (1, lowest; 4 , highest) as rated by the participants were 2.89 (SD, 0.4) for emotional distracters and 1.25 (SD, 0.23) for neutral distracters. Pairwise comparison of the rating scores was highly significant $\left(T_{(14)}=\right.$ 11.33; $p<0.0001$ ), thus confirming that the subjects' rating scores were consistent with the normative scores (see Materials and Methods). The average scores for distractibility were 2.71 $(\mathrm{SD}=0.46)$, for emotional distracters and $1.45(\mathrm{SD}=0.34)$ for neutral distracters. Their pairwise comparison was also highly significant $\left(T_{(14)}=8.30 ; p<0.0001\right)$, thus confirming that participants perceived the emotional pictures as more distracting than the neutral pictures.

\section{Working memory performance}

Confirming our first prediction, emotional distracters produced the most detrimental effect on working memory performance (Fig. 2A). Participants correctly identified probes that were part of the memoranda (Hits) on $75 \%(\mathrm{SD}=14)$ of the trials for the emotional condition, $80 \%(\mathrm{SD}=10)$ of the trials for the neutral condition, and $88 \%(\mathrm{SD}=8)$ of the trials for the no-distraction control condition (i.e., the scrambled pictures). An ANOVA yielded a significant main effect of distracter type $\left(F_{(2,14)}=9.30\right.$; $p<0.0008$ ), and all planned paired comparisons were significant ( $p<0.05$ for all). These results were confirmed when statistics were computed on the corrected recognition scores (hits - false alarms; $\left.F_{(2,14)}=13.77 ; p<0.0001\right)$ and on the averaged scores for correct responses (hits and correct rejections; $F_{(2,14)}=13.35 ; p<$ $0.0001)$; all planned paired comparisons were significant $(p<$ $0.05)$.

\section{fMRI results}

Differential patterns of activity in the dorsal executive and ventral affective neural systems

Confirming our second prediction, emotional distracters produced markedly different patterns of activity in the dorsal executive and ventral emotional neural systems during the working memory delay period (Figs. $2 B, C, 3$; Table 1 ). As expected, when compared with both neutral and scrambled distracters, the emotional distracters evoked stronger activity in ventral regions involved in affective processing, including the amygdala, vlPFC,
B. dIPFC Activity

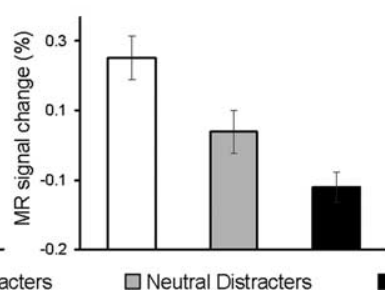

Neutral Distracters
C. Amygdala Activity

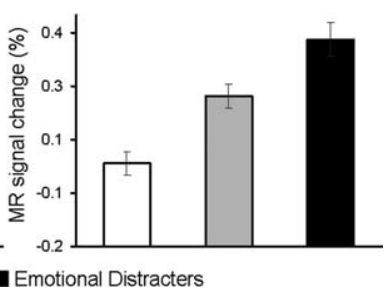

Scrambled Distracters

Figure 2. Brain-behavior relationship showing the direct link between brain activity and memory performance. $A$, As illusty during the delay period in brain regions typically associated with active maintenance of task-relevant information in typically associated with emotional processing (e.g., amygdala). Hits, Correctly identified old probes; FAs, incorrectly identified ars represent $S E M$.

and medial PFC. Moreover, when compared with both neutral and scrambled distracters, emotional distracters simultaneously evoked strong deactivation in dorsal executive brain regions, including the dlPFC, LPC, and posterior cingulate cortex, and reduced the brain activity during the delay period in these regions to below prestimulus baseline levels (Fig. 3).

The statistical significance of these contrasting patterns of activity was assessed by repeated-measures ANOVAs computed on the percentage change in the MR signal from regions of interest traced around the peak active voxels for the comparisons of interest. Analyses reported in this section compared the brain activity observed at time points within the $10-16 \mathrm{~s}$ period after memorandum onset, when the differential effects of the distraction conditions on activity during the delay period were most evident (Fig. 3, Table 1), and are based on data averaged across the two hemispheres. A two-way 2 (neural system, dorsal vs ventral) $\times 3$ (distracter, emotional vs neutral vs scrambled) ANOVA computed on the MR signal extracted from dorsal (dlPFC and LPC) and ventral (amygdala and the vlPFC) regions yielded a highly significant neural system $\times$ distracter interaction $\left(F_{(2,28)}=133.18 ; p<0.0001\right)$. One-way ANOVAs computed on the percentage change in the MR signal for each distracters type (emotional vs neutral vs scrambled) yielded significant main effects in all four brain regions [dlPFC, $F_{(2,14)}=39.66, p<0.0001$; LPC, $F_{(2,14)}=40.75, p<0.0001$; vlPFC, $F_{(2,14)}=36.37, p<$ 0.0001 ; amygdala, $\left.F_{(2,14)}=52.25, p<0.0001\right)$. Post hoc analyses confirmed that the emotional distracters produced greater effects (i.e., greater deactivation in the dorsal system and greater activation in the ventral system) than either neutral or scrambled distracters in each region ( $p<0.01$ for both). Additional support for this claim was derived from a 2 (neural system, dorsal vs ventral) $\times 2$ (distracter, emotional vs neutral) ANOVA, which also yielded a significant neural system $\times$ distracter interaction $\left(F_{(1,14)}=59.66 ; p<0.0001\right)$. These analyses confirmed that the interaction was attributable to emotion rather than to the presence of meaningful stimuli per se and provide clear evidence that impaired WM performance in the presence of emotional distracters is linked to contrasting patterns of activity in the dorsal and ventral neural systems.

To further investigate whether impaired WM performance in the presence of emotional distraction is related to reduced activity during the delay period in the dorsal executive system, additional analyses were conducted to identify regions within the dorsal system in which activity during the delay period differentiated correct trials from incorrect trials. Once identified, we then 


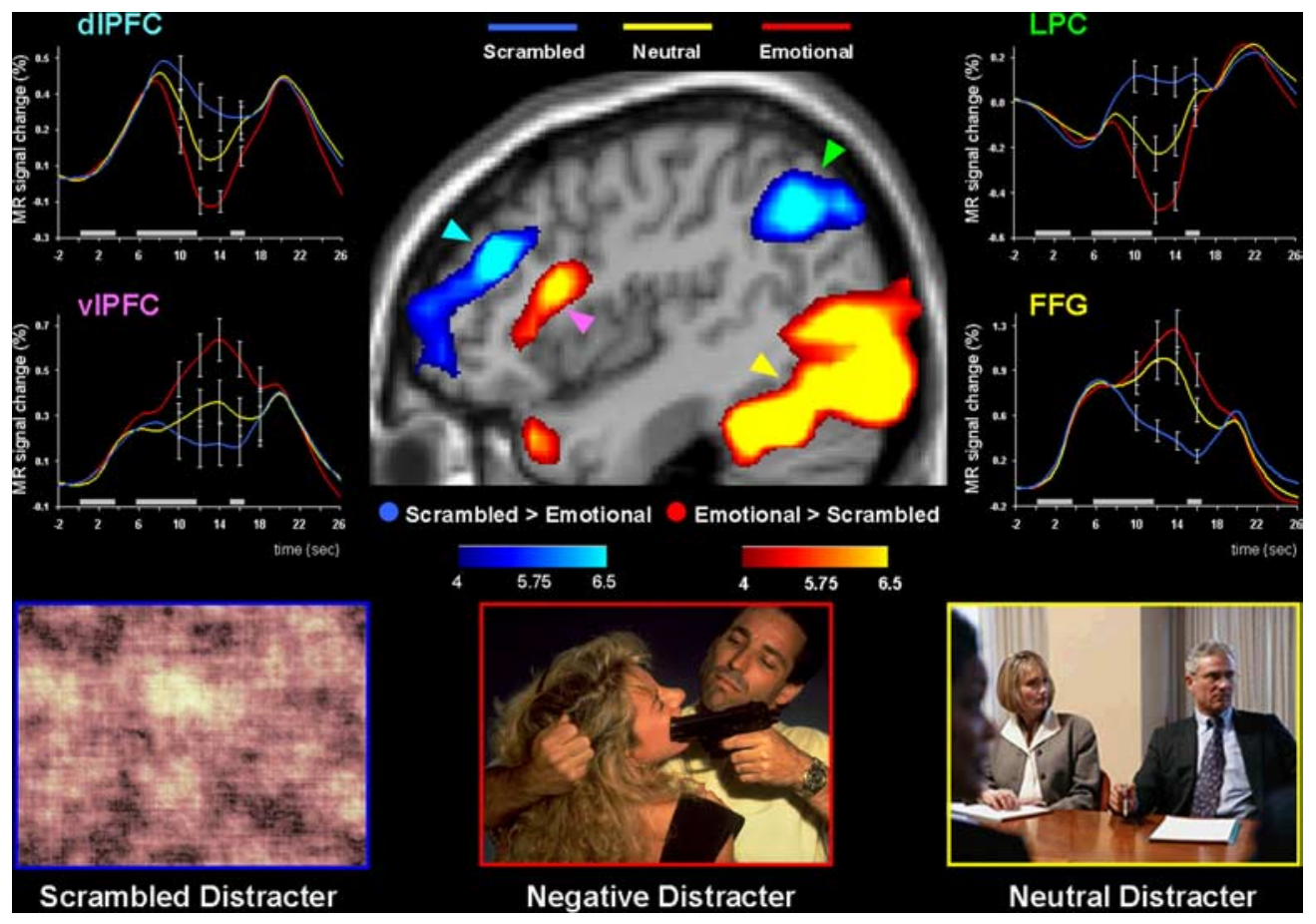

Figure 3. Dorsoventral dissociable patterns of activity in the presence of emotional distracters. Emotional distracters produced the most disrupting effect on the activity during the delay period in a set of dorsal brain regions associated with cold executive processes (the blue blobs) while producing the most enhancing effect on activity in a set of ventral brain regions associated with hot emotional processing (the red blobs). The central image shows activation maps of the direct contrasts between the most versus least distracting conditions (i.e., emotional vs scrambled), superimposed on a high-resolution brain image displayed in a lateral view of the right hemisphere. The colored horizontal bars at the bottom of the brain image indicate the gradients of the $t$ values for the activation maps displayed. The line graphs show the time courses of activity in representative dorsal and ventral brain regions (indicated by color-coded arrows). The gray rectangular boxes above the $x$-axes indicate the onset and duration of the memoranda, distracters, and probes, respectively. FFG, Fusiform gyrus.

Table 1. Differential effect of emotional distracters in dorsal executive versus ventral affective neural systems

\begin{tabular}{|c|c|c|c|c|c|c|c|c|c|}
\hline \multirow[b]{2}{*}{ Neural system } & \multirow[b]{2}{*}{ Brodmann's area } & \multirow[b]{2}{*}{ Side } & \multicolumn{3}{|c|}{ Talairach coordinates } & \multicolumn{3}{|c|}{$t$ values of paired contrasts } & \multirow[b]{2}{*}{ Time $(s)$} \\
\hline & & & $x$ & $y$ & $Z$ & Scram $>$ Emo & Scram $>$ Neu & $\mathrm{Neu}>$ Emo & \\
\hline \multicolumn{10}{|l|}{ Dorsal executive } \\
\hline \multirow{2}{*}{ dIPFC } & $9 / 46$ & $\mathrm{R}$ & 46 & 38 & 22 & 9.6 & 6.5 & 4.3 & 12 \\
\hline & 9 & $\mathrm{~L}$ & -34 & 29 & 26 & 5.1 & 5.4 & & 12 \\
\hline \multirow[t]{2}{*}{$\mathrm{LPC}$} & 40 & $\mathrm{R}$ & 46 & -54 & 40 & 9.7 & 5.6 & 5.4 & 12 \\
\hline & 40 & $\mathrm{~L}$ & -53 & -54 & 43 & 5.3 & 5.1 & & 12 \\
\hline $\mathrm{pCG}$ & 31 & M & 4 & -29 & 35 & 5.6 & 4.1 & & 12 \\
\hline $\mathrm{BG}$ & cn & $\mathrm{R}$ & 18 & 24 & 4 & 5.8 & 4.2 & $3.1^{a}$ & 12 \\
\hline \multicolumn{10}{|l|}{ Ventral affective } \\
\hline \multirow[t]{2}{*}{ vIPFC } & 45 & $\mathrm{R}$ & 53 & 28 & 12 & 9.1 & 9.4 & 4.0 & 14 \\
\hline & $45 / 47$ & $\mathrm{~L}$ & -50 & 27 & 4 & 5.4 & 4.6 & & 16 \\
\hline $\operatorname{sgCG}$ & 25 & M & 2 & 13 & -16 & 6.2 & 4.2 & & 14 \\
\hline Insula & 13 & $\mathrm{R}$ & 40 & -12 & -1 & 5.1 & 3.8 & & 14 \\
\hline \multirow[t]{2}{*}{ Amygdala } & & $\mathrm{R}$ & 22 & -1 & -12 & 10.0 & 6.0 & 3.8 & 16 \\
\hline & & $\mathrm{L}$ & -22 & -4 & -12 & 7.7 & 7.5 & $3.0^{a}$ & 16 \\
\hline \multirow[t]{2}{*}{$\mathrm{HC}$} & & $\mathrm{R}$ & 28 & -14 & -11 & 5.6 & 4.6 & 4.9 & 16 \\
\hline & & $\mathrm{L}$ & -24 & -12 & -8 & 7.6 & 4.6 & 5.3 & 16 \\
\hline Hthal & & M & 0 & 0 & -7 & 10.0 & 5.0 & & 14 \\
\hline Midbrain & & $M$ & 2 & -15 & 3 & 6.9 & 7.4 & & 12 \\
\hline
\end{tabular}

$t$ values of paired contrasts between the conditions of interest as a result of whole-brain voxel-based analyses $(p<0.001 ; t>3.79)$ are shown for typical dorsal executive and ventral affective brain regions. The $x, y, z$ coordinates denote the location of the peak voxel in Talairach space (Talairach and Tournoux, 1988). The effects identified during the delay interval typically peaked between 12 and $16 \mathrm{~s}$ after the onset of the memoranda (TRs, 6 - 8 after onset). Emo, Emotional; Neu, neutral; Scram, scrambled; R, right; L, left; M, middle; $p C G$, posterior cingulated gyrus; BG, basal ganglia; cn, caudate nucleus; sgCG, subgenual cingulate gyrus; $H C$, hippocampus; Hthal, hypothalamus.

${ }^{a}$ Significant at $p<0.005(t>2.97)$.

examined the influence of emotional distraction for this region. Brain activity for trials associated with correct (hits and correct rejections) responses was compared with activity associated with incorrect responses (misses and false alarms) (supplemental material, available at www.jneurosci.org). Based on evidence from previous WM studies (Sakai et al., 2002), we predicted that regions within dIPFC would show greater activity in the delay pe- riod for the correct than for the incorrect trials. We further predicted that the incorrect trials for the emotional distraction condition (the condition associated with the lowest level of performance) would evoke the lowest level of activity in these regions.

Both predictions were confirmed. A region in the dIPFC (BA9; Talairach coordinates: $x, y, z=-32,40,31 ; T_{(9)}=4.27 ; p<$ 
0.001) was identified that exhibited greater activity during the delay period for the correct than for the incorrect trials. Moreover, the emotional trials associated with incorrect responses produced the lowest level of activity during the delay period in this region (supplemental Fig. 1, available at www.jneurosci.org as supplemental material). These findings provide further support for the idea that activity during the delay period in the dorsal executive neural system predicts WM performance, and that this activity is susceptible to interfering effects from emotional distracters presented during the delay interval.

\section{Hemispheric asymmetry in the} processing of emotional distracters Consistent with the role of the right hemisphere in the processing of negative emotions (Davidson, 1995; Borod et al., 1998), we also found that emotional distracters had a greater impact on the brain activity during the delay period in specific regions in the right hemisphere than in the left hemisphere, and this effect was more pronounced in the prefrontal regions (Fig. 4). Supporting this idea, two-way ANOVAs including hemisphere (left vs right) and distracter (emotional vs neutral vs scrambled) as factors yielded significant hemisphere $\times$ distracter interactions in the $\operatorname{dlPFC}\left(F_{(2,28)}=13.37 ; p<0.0001\right)$, vlPFC $\left(F_{(2,28)}=18.62 ; p<0.0001\right)$, and the LPC $\left(F_{(2,28)}=5.9 ; p<0.02\right)$. Notably, the most significant effects were found in the prefrontal regions, dlPFC and vlPFC, and post hoc analyses confirmed that these interactions were attributable to the fact that negative distracters had a greater impact on the activity during the delay period in the right hemisphere than in the left hemisphere. Although separate investigation of activity in these regions revealed significant main effects of distraction (emotional vs neutral vs scrambled) in both hemispheres and regions (left dlPFC, $F_{(2,14)}=$ 25.07, $p<0.0001$; left vlPFC, $F_{(2,14)}=10.63, p<0.0005$; right dlPFC, $F_{(2,14)}=37.14, p<0.0001$; right vlPFC, $F_{(2,14)}=48.55$, $p<0.0001)$, post hoc analyses showed that the effect size between the emotional and the neutral distracters was greater in the right hemisphere (dlPFC, $p<0.0005$; vlPFC, $p<0.0001$ ) than in the left hemisphere dIPFC, $p>0.47$; vlPFC, $p<0.001$ ), and that this hemispheric asymmetry was more pronounced in the dlPFC. These findings were confirmed by 2 (hemisphere, left vs right) $\times$ 2 (distracter, emotional vs neutral) ANOVAs, which also yielded significant hemisphere $\times$ distracter interactions both in the $\operatorname{dlPFC}\left(F_{(1,14)}=14.95 ; p<0.005\right)$ and in the $\operatorname{vlPFC}\left(F_{(1,14)}=\right.$ 5.47; $p<0.05)$. Finally, post hoc analyses confirmed that these interactions were attributable to the fact that the difference between the emotional and neutral distracters tended to be more significant in the right hemisphere (dlPFC, $p<0.001$; vlPFC, $p<$
0.0001) than in the left hemisphere (dlPFC, $p>0.48$; vlPFC, $p<$ $0.002)$.

\section{Individual differences in the processing of} emotional distracters

The present study also identified individual differences concerning the relationship between brain activity and behavioral responses in the presence of emotional distraction. Specifically, consistent with the role of the vlPFC in inhibitory processes (Jonides et al., 1998; D’Esposito et al., 1999; Smith and Jonides, 1999), activity in this region was highly correlated with the subjects' overall distractibility scores for the emotional $(r=-0.74$; $p<0.001)$ but not for the neutral $(r=0.13 ; p>0.65)$ distracters (Fig. 5) (see Materials and Methods). Moreover, activity in the same vlPFC region that correlated with the subjective distracti- 
bility scores (BA45; Talairach coordinates: $x, y, z=55,24,15$ ) also correlated with the subjective emotional ratings for emotional $(r=-0.73 ; p<0.001)$ but not for the neutral $(r=0.34$; $p>0.1)$ distracters. Subjects who showed greater activity to emotional distracters in the vlPFC also tended to rate emotional distracters as both less distracting and less emotional. These results were consistent with the findings that individual distractibility and emotional indices were significantly correlated with each other for the emotional $(r=0.72 ; p<0.002)$ but not for the neutral $(r=0.22 ; p>0.25)$ distracters. Finally, correlation analyses also showed that activity in the vlPFC was also correlated with indices of relative impact of the meaningful distraction on WM performance, which were calculated by subtracting the average of correct responses in the presence of meaningful distracters (both emotional and neutral) from the average of correct responses in the presence of meaningless distraction (i.e., scrambled distracters). These analyses identified a subregion of the inferior frontal gyrus (BA9; $x, y, z=40,9,25)$, which was correlated with the indices of relative impact of the meaningful distraction on WM performance for both emotional $(r=-0.58$; $p<$ $0.02)$ and neutral $(r=-0.57 ; p<0.02)$ distracters. Collectively, these findings provide evidence for a role of the vlPFC in general inhibitory processes and for a specific involvement of this region in the inhibition of distracting emotions.

\section{Discussion}

Perhaps as a consequence of their potential as cues for threats or danger, emotional stimuli have salience that transcends current goal-directed behavior. Regardless of their relevance to ongoing activities, emotional stimuli can capture and divert attention. The enhanced significance of emotional stimuli can benefit some cognitive functions (e.g., better memory for emotional events) but can also cause detrimental effects on ongoing behavior (e.g., increased distractibility), particularly if emotional stimuli are task irrelevant (e.g., passing the scene of a tragic accident while driving). Although the enhancing effects of emotion on cognitive functions has been the focus of intensive research (Christianson, 1992; Dolcos et al., 2004b; Kensinger and Corkin, 2004; McGaugh, 2004; Phelps, 2004; Sharot et al., 2004; Dolcos et al., 2005, 2006), the detrimental effects of emotion on cognitive functions have received much less attention (Johnson et al., 2005; Miu et al., 2005; Most et al., 2005) (but see Seibert and Ellis, 1991; Oaksford et al., 1996).

The present fMRI study investigated the neural mechanisms underlying the detrimental effect of goal-irrelevant emotional distracters on the ability to maintain goal-relevant information into WM. Our results demonstrate a markedly different pattern of activation evoked by the emotional distracters, which strongly activated ventral system structures involved in emotional processing while dramatically disrupting delay interval activity in dorsal brain regions previously implicated in active maintenance of task-relevant information in WM. Importantly, this disruption of dorsal system activation was associated with impaired memory performance.

These differential patterns of activity are consistent with evidence separately linking the dorsal regions with WM operations and processing associated with distraction and the ventral regions with aspects of emotional processing. The pattern of activation observed in the dorsal regions (e.g., dlPFC and the LPC) is consistent with evidence associating these brain regions with WM maintenance (Courtney et al., 1997; Fuster, 1997; Smith and Jonides, 1999; Chafee and Goldman-Rakic, 2000), with the inhibition of task-irrelevant information (Mishkin and Manning,
1978; Funahashi et al., 1993; Chao and Knight, 1998; Shimamura, 2000) and with protection against distraction (Jiang et al., 2000; Sakai et al., 2002; Jha et al., 2004). The pattern of activation observed in the ventral regions (e.g., the amygdala and the vlPFC) is consistent with abundant evidence associating these regions with various aspects of emotional processing (Davidson and Irwin, 1999; Phan et al., 2002).

The novel aspect of the present study is the interaction observed between the dorsal and ventral systems and the effect of that interaction on behavior. Unlike the present study, previous functional neuroimaging studies of WM with distraction either did not involve emotional distracters (Jiang et al., 2000; Sakai et al., 2002; Jha et al., 2004; Yoon et al., 2006) or did not link changes in brain activity to impaired behavioral performance in the presence of emotional distracters (Perlstein et al., 2002; Yamasaki et al., 2002; Wang et al., 2005). For instance, previous studies from our group using target detection (or, oddball) tasks reported that infrequent and irregularly presented emotional distracters activated the amygdala, ventrolateral frontal, and ventromedial frontal cortices while simultaneously evoking negative activation (or deactivation) of dlPFC (Yamasaki et al., 2002; Wang et al., 2005). These negative activations, or deactivations, were manifested as a change below prestimulus baseline of the blood oxygen leveldependent (BOLD) MRI signal. However, as these negative activations in dlPFC were observed in the absence of a behavioral consequence, the functional significance of the interactions between the dorsal and ventral systems was undetermined.

The present results extends the existing evidence by showing for the first time that the contrasting patterns of activity in the dorsal and ventral brain systems are directly linked to changes in behavioral performance. Consistent with our previous studies (Yamasaki et al., 2002; Wang et al., 2005), the presentation of an emotional distracter in the present study caused a relative negative activation; that is, the sustained positive BOLD signal that normally occurs during the delay period and maintenance interval of a WM task in the absence of distraction was driven below prestimulus baseline by the emotional distracter. Unlike our previous studies, this disruption of sustained delay interval activity was associated with impaired WM memory performance. These findings complement our previous studies (Yamasaki et al., 2002; Wang et al., 2005) and provide strong support for the idea derived from clinical studies that activity in the hot emotional and the cold executive brain regions are interconnected such that increased activity in the ventral emotional system is associated with decreased activity in the dorsal executive regions (Dolan et al., 1993; Drevets and Raichle, 1998; Mayberg et al., 1999), which results in impaired cognitive performance. These findings provide strong support for the idea that activation of the ventral system in the presence of transient emotional distracters temporarily takes off-line the dorsal system and impairs cognitive performance.

The right-lateralization pattern observed in the present study is different from the left-lateralized pattern identified in female participants by studies of emotional memory encoding (Cahill et al., 2001) but is consistent with current models of hemispheric asymmetry in emotional processing that associate negative emotions with the right hemisphere (Davidson, 1995; Borod et al., 1998). Supporting these models, neuropsychological studies of frontal lobe patients have shown that patients with left hemisphere lesions tend to experience negative emotions such as sadness (Morris et al., 1996), and functional neuroimaging studies of nonclinical participants have identified regions of the right hemisphere that are particularly associated with processing of negative 
emotions (Dolcos et al., 2004a). Consistent with the suggestion that hemispheric lateralization in emotional processing is more pronounced in the prefrontal regions (Davidson, 1995), Dolcos et al. (2004a) recently identified a region in the right vlPFC that was particularly sensitive to transient processing of negative pictorial stimuli. Interestingly, that region overlaps with the vlPFC region that showed right-lateralized hemispheric asymmetry in the processing of negative distracters in the present study. It would be interesting to investigate whether an opposite pattern of lateralization is present in male participants during processing of transient emotional distracters, similar to the findings from studies of emotional memory encoding (Cahill et al., 2001).

Finally, the individual differences identified in the present study suggest that the right vlPFC is involved in the inhibition of emotional distraction. Given that previous studies of inhibition (Jonides et al., 1998; D’Esposito et al., 1999; Smith and Jonides, 1999) have associated the left vlPFC with inhibitory processes and that studies of emotion have associated the right vlPFC with the processing of transient negative emotions (Phan et al., 2002; Dolcos et al., 2004a), it is possible that activity in the right vlPFC identified in the present study reflects "pure" emotional processing rather than inhibitory processes. However, studies that have specifically associated the right vlPFC with the inhibition of negative emotions (Petrovic et al., 2002; Ochsner et al., 2004) suggest that greater activity in this region reflects enhanced inhibitory processes engaged to control the impact of distracting negative emotions. The present correlation results provide evidence consistent with the latter interpretation by showing that subjects showing greater activity in this region to negative distracters also tended to rate them as both less emotional and less distracting, possibly as a result of engaging inhibitory processes that diminished the subjective experience of being distracted. Activity in a different subregion of the inferior frontal gyrus also correlated with the WM memory performance, but this region did not dissociate the impact of emotional and neutral distracters. Collectively, these findings suggest a functional dissociation within the vlPFC with respect to its involvement in controlling the subjective feeling of being distracted as opposed to controlling the actual impact of emotional distraction on cognitive performance. These findings are consistent with the evidence supporting a general role of the vlPFC in inhibitory processes (Aron et al., 2004) and identify a specific role of this region in the inhibition of distracting emotions.

In summary, the present study provides the first functional neuroimaging evidence that impaired performance in the presence of emotional distracters is associated with disrupted activity in the brain regions responsible for active maintenance of goalrelevant information in WM (dorsal system) and enhanced activity in the brain regions responsible for emotional processing (ventral system). Consistent with models of hemispheric asymmetry in emotional processing, the present study also provides evidence for a right-lateralization of the observed effects. Finally, the present study also identified a specific role of the right vlPFC in the inhibition of distracting emotions. These results shed light on the neural mechanisms underlying the impairing effect of emotional distraction on working memory and extend the evidence concerning the neural mechanisms underlying the modulatory effect of emotion on executive functions (Gray et al., 2002; Perlstein et al., 2002; Yamasaki et al., 2002; Bishop et al., 2004; Simon-Thomas et al., 2005; Wang et al., 2005; Mather et al., 2006). The present findings provide the first direct evidence that the detrimental effect of emotional distracters on working memory maintenance is associated with cognitive-affective interac- tions that involve interplays between a dorsal neural system associated with cold executive processing and a ventral system associated with hot emotional processing.

\section{References}

Aron AR, Robbins TW, Poldrack RA (2004) Inhibition and the right inferior frontal cortex. Trends Cogn Sci 8:170-177.

Baddeley AD (1986) Working memory. Oxford: Oxford UP.

Bishop S, Duncan J, Brett M, Lawrence AD (2004) Prefrontal cortical function and anxiety: controlling attention to threat-related stimuli. Nat Neurosci 7:184-188.

Borod JC, Obler LK, Erhan HM, Grunwald IS, Cicero BA, Welkowitz J, Santschi C, Agosti RM, Whalen JR (1998) Right hemisphere emotional perception: evidence across multiple channels. Neuropsychology 12:446-458.

Cahill L, Haier RJ, White NS, Fallon J, Kilpatrick L, Lawrence C, Potkin SG, Alkire MT (2001) Sex-related difference in amygdala activity during emotionally influenced memory storage. Neurobiol Learn Mem 75:1-9.

Chafee MV, Goldman-Rakic PS (2000) Inactivation of parietal and prefrontal cortex reveals interdependence of neural activity during memoryguided saccades. J Neurophysiol 83:1550-1566.

Chao LL, Knight RT (1998) Contribution of human prefrontal cortex to delay performance. J Cogn Neurosci 10:167-177.

Christianson S- $\AA$ (1992) The handbook of emotion and memory: research and theory. Hillsdale, NJ: Lawrence Erlbaum Associates.

Courtney SM, Ungerleider LG, Keil K, Haxby JV (1997) Transient and sustained activity in a distributed neural system for human working memory. Nature 386:608-611.

Davidson RJ (1995) Cerebral asymmetry, emotion and affective style. In: Brain asymmetry (Davidson RJ, Hugdahl K, eds), pp 361-387. Cambridge, MA: MIT.

Davidson RJ, Irwin W (1999) The functional neuroanatomy of emotion and affective style. Trends Cogn Sci 3:11-20.

Davis M, Whalen PJ (2001) The amygdala: vigilance and emotion. Mol Psychiatry 6:13-34.

D’Esposito M, Postle BR, Jonides J, Smith EE (1999) The neural substrate and temporal dynamics of interference effects in working memory as revealed by event-related functional MRI. Proc Natl Acad Sci USA 96:7514-7519.

Dolan RJ, Bench CJ, Liddle PF, Friston KJ, Frith CD, Grasby PM, Frackowiak RS (1993) Dorsolateral prefrontal cortex dysfunction in the major psychoses: symptom or disease specificity? J Neurol Neurosurg Psychiatry 56:1290-1294

Dolcos F, LaBar KS, Cabeza R (2004a) Dissociable effects of arousal and valence on prefrontal activity indexing emotional evaluation and subsequent memory: an event-related fMRI study. NeuroImage 23:64-74.

Dolcos F, LaBar KS, Cabeza R (2004b) Interaction between the amygdala and the medial temporal lobe memory system predicts better memory for emotional events. Neuron 42:855-863.

Dolcos F, LaBar KS, Cabeza R (2005) Remembering one year later: role of the amygdala and the medial temporal lobe memory system in retrieving emotional memories. Proc Natl Acad Sci USA 102:2626-2631.

Dolcos F, LaBar KS, Cabeza R (2006) The memory-enhancing effect of emotion: functional neuroimaging evidence. In: Memory and emotion: interdisciplinary perspectives (Uttl B, Ohta N, Siegenthaler AL, eds). Oxford: Blackwell, in press.

Drevets WC, Raichle ME (1998) Reciprocal suppression of regional cerebral blood flow during emotional versus higher cognitive processes: implications for interactions between emotion and cognition. Cogn Emotion 12:353-385.

Ellis HC, Ashbrook PW (1988) Resource allocation model of the effects of depressed mood states on memory. In: Affect, cognition, and social behavior: new evidence and integrative attempts (Fiedler K, Forgas J, eds), pp 25-43. Toronto: Hogrefe.

Funahashi S, Bruce CJ, Goldman-Rakic PS (1993) Dorsolateral prefrontal lesions and oculomotor delayed-response performance: evidence for mnemonic "scotomas." J Neurosci 13:1479-1497.

Fuster JM (1997) Network memory. Trends Neurosci 20:451-459.

Gray JR, Braver TS, Raichle ME (2002) Integration of emotion and cognition in the lateral prefrontal cortex. Proc Natl Acad Sci USA 99:4115-4120. 
Jha AP, Fabian SA, Aguirre GK (2004) The role of prefrontal cortex in resolving distractor interference. Cogn Affect Behav Neurosci 4:517-527.

Jiang Y, Haxby JV, Martin A, Ungerleider LG, Parasuraman R (2000) Complementary neural mechanisms for tracking items in human working memory. Science 287:643-646.

Johnson MK, Raye CL, Mitchell KJ, Greene EJ, Cunningham WA, Sanislow CA (2005) Using fMRI to investigate a component process of reflection: prefrontal correlates of refreshing a just-activated representation. Cogn Affect Behav Neurosci 5:339-361.

Jonides J, Smith EE, Marshuetz C, Koeppe RA, Reuter-Lorenz PA (1998) Inhibition in verbal working memory revealed by brain activation. Proc Natl Acad Sci USA 95:8410-8413.

Kensinger EA, Corkin S (2004) Two routes to emotional memory: distinct neural processes for valence and arousal. Proc Natl Acad Sci USA 101:3310-3315.

Lang PJ, Greenwald MK, Bradley MM, Hamm AO (1993) Looking at pictures: affective, facial, visceral, and behavioral reactions. Psychophysiology 30:261-273.

Lang PJ, Bradley MM, Cuthberg BN (1997) International affective picture system. Gainesville, FL: NIMH Center for the Study of Emotion and Attention.

Mather M, Mitchell KJ, Raye CL, Novak DL, Greene EJ, Johnson MK (2006) Emotional arousal can impair feature binding in working memory. J Cogn Neurosci, in press.

Mayberg HS (1997) Limbic-cortical dysregulation: a proposed model of depression. J Neuropsychiatry 9:471-481.

Mayberg HS, Liotti M, Brannan SK, McGinnis S, Mahurin RK, Jerabek PA, Silva JA, Tekell JL, Martin CC, Lancaster JL, Fox PT (1999) Reciprocal limbic-cortical function and negative mood: converging PET findings in depression and normal sadness. Am J Psychiatry 156:675-682.

McGaugh JL (2004) The amygdala modulates the consolidation of memories of emotionally arousing experiences. Annu Rev Neurosci 27:1-28.

Mishkin M, Manning FJ (1978) Non-spatial memory after selective prefrontal lesions in monkeys. Brain Res 143:313-323.

Miu AC, Heilman RM, Opre A, Miclea M (2005) Emotion-induced retrograde amnesia and trait anxiety. J Exp Psychol Learn Mem Cogn 31:1250 1257.

Morris PL, Robinson RG, Raphael B, Hopwood MJ (1996) Lesion location and poststroke depression. J Neuropsychiatry Clin Neurosci 8:399-403.

Most SB, Chun MM, Widders DM, Zald DH (2005) Attentional rubbernecking: cognitive control and personality in emotion-induced blindness. Psychon Bull Rev 12:654-661.

Oaksford M, Grainger B, Morris F, Williams JMG (1996) Mood, reasoning, and central executive processes. J Exp Psychol 22:476-492.

Ochsner KN, Ray RD, Cooper JC, Robertson ER, Chopra S, Gabrieli JD, Gross
JJ (2004) For better or for worse: neural systems supporting the cognitive down- and up-regulation of negative emotion. NeuroImage 23:483-499.

Perlstein WM, Elbert T, Stenger VA (2002) Dissociation in human prefrontal cortex of affective influences on working memory-related activity. Proc Natl Acad Sci USA 99:1736-1741.

Petrovic P, Kalso E, Petersson KM, Ingvar M (2002) Placebo and opioid analgesia-imaging a shared neuronal network. Science 295:1737-1740.

Phan KL, Wager T, Taylor SF, Liberzon I (2002) Functional neuroanatomy of emotion: a meta-analysis of emotion activation studies in PET and fMRI. NeuroImage 16:331-348.

Phelps EA (2004) Human emotion and memory: interactions of the amygdala and hippocampal complex. Curr Opin Neurobiol 14:198-202.

Sakai K, Rowe JB, Passingham RE (2002) Active maintenance in prefrontal area 46 creates distractor-resistant memory. Nat Neurosci 5:479-484.

Seibert PS, Ellis HC (1991) Irrelevant thoughts, emotional mood states, and cognitive task performance. Mem Cogn 19:507-513.

Sharot T, Delgado MR, Phelps EA (2004) How emotion enhances the feeling of remembering. Nat Neurosci 7:1376-1380.

Shields SA (1991) Gender in the psychology of emotion: a selective research review. In: International review of studies on emotion (Strongman KT, ed), pp 227-245. New York: Wiley.

Shimamura AP (2000) The role of the prefrontal cortex in dynamic filtering. Psychobiology 28:207-218.

Simon-Thomas ER, Role KO, Knight RT (2005) Behavioral and electrophysiological evidence of a right hemisphere bias for the influence of negative emotion on higher cognition. J Cogn Neurosci 17:518-529.

Smith EE, Jonides J (1999) Storage and executive processes in the frontal lobes. Science 283:1657-1661.

Talairach J, Tournoux P (1988) A co-planar sterotactic atlas of the human brain. Stuttgart, Germany: Thieme.

Wang L, McCarthy G, Song AW, Labar KS (2005) Amygdala activation to sad pictures during high-field (4 tesla) functional magnetic resonance imaging. Emotion 5:12-22.

Watts FN, MacLeod AK, Morris L (1988) Associations between phenomenal and objective aspects of concentration problems in depressed patients. Br J Psychol 79:241-250.

Yamasaki H, LaBar KS, McCarthy G (2002) Dissociable prefrontal brain systems for attention and emotion. Proc Natl Acad Sci USA 99:11447-11451.

Yoon JH, Curtis CE, D’Esposito M (2006) Differential effects of distraction during working memory on delay-period activity in the prefrontal cortex and the visual association cortex. NeuroImage, in press.

Zald DH (2003) The human amygdala and the emotional evaluation of sensory stimuli. Brain Res Brain Res Rev 41:88-123. 